\title{
Expression of Circulating Plasma Long Non-Coding RNA uc063iod.1 and Its Relationship with Coronary Artery Disease
}

\author{
Vaidya Rajan1, Genshan Ma2*, Hong Jin² \\ ${ }^{1}$ School of Medicine, Southeast University, Nanjing, China \\ ${ }^{2}$ Department of Cardiology, Zhongda Hospital, Southeast University, Nanjing, China \\ Email: ^magenshan@hotmail.com
}

How to cite this paper: Rajan, V., Ma, G.S. and Jin, H. (2018) Expression of Circulating Plasma Long Non-Coding RNA uc063iod.1 and Its Relationship with Coronary Artery Disease. Open Journal of Internal Medicine, 8, 131-145.

https://doi.org/10.4236/ojim.2018.82014

Received: February 24, 2018

Accepted: April 25, 2018

Published: April 28, 2018

Copyright $\odot 2018$ by authors and Scientific Research Publishing Inc. This work is licensed under the Creative Commons Attribution International License (CC BY 4.0).

http://creativecommons.org/licenses/by/4.0/

\begin{abstract}
Objective: To investigate the expression of LncRNA uc063iod.1 in patients with different diseases and to explore the correlation between expression level of LncRNA uc063iod.1 and Coronary Artery Disease (CAD). Method: Plasma samples were prepared using the blood extracted from total 101 patients undergoing coronary artery angiogram (63 CAD patients and 38 non CAD patients). The expression level of LncRNA uc063iod.1 was detected using quantitative real time polymerase chain reaction (qRT-PCR) and the relationship between expression level of LncRNA uc063iod.1 and CAD was explored. The clinicopathological features of patients with CAD were also discussed in our study. Results: The expression of LncRNA uc063iod.1 was significantly increased in the plasma of patients with CAD $(\mathrm{p}<0.05)$. AUC was 0.701 (95\%CI: $0.598-0.804$ ), and the sensitivity and specificity were $77.8 \%$ and $57.9 \%$ respectively. Conclusion: Upregulation of LncRNA uc063iod.1 in plasma can be used as a biomarker for the diagnosis of CAD.
\end{abstract}

\section{Keywords}

LncRNA uc063iod.1, LncRNA E330013P06, Coronary Artery Disease, Plasma, qRT-PCR

\section{Introduction}

Cardiovascular diseases (CVD) are the leading cause of morbidity and mortality in the world. An estimated 17.7 million people died from CVDs in 2015, representing $31 \%$ of all global deaths. Of these deaths, an estimated 7.4 million were 
due to Coronary artery Disease (CAD). Atherosclerotic disease and its thrombotic complication are the common manifestation of CVDs that leads to the development of CAD; if untreated, it progresses into Myocardial Infarction and Heart Failure [1]. Reducing lipids, antiplatelet agents, anticoagulants, Percutaneous Coronary Intervention (PCI), and Coronary Artery Bypass Graft (CABG) surgery are available effective treatment measures for CAD. However, those treatments are challenged by the adverse effects of drugs, drug tolerance and high recurrence rate, resulting no change in mortality. Therefore, the development of a new non-invasive method that can diagnose CAD early in its onset is critical in improving its prognosis.

Modern advancement in genome-wide analyses has identified large number of long non-coding RNAs (LncRNAs), which are transcribed in human genome. LncRNAs are non-protein coding RNA, which are 200 to 10,000 nucleotides in length [2]. Even though LncRNAs are new to the research world, they have already been used as promising biomarkers for diagnosis of several diseases. LncRNA PCA3 is already a well-established biomarker for prostate cancer [3]. Likewise, LncRNA ATB can serve as biomarker to detect Coal Worker Pneumoconiosis [4]. And LncRNA LIPCAR is shown to have potential to serve as a biomarker for heart failure [5]. Recent studies have already claimed several LncRNAs are associated with initiation and progression of coronary heart diseases [6].

LncRNA uc063iod.1 is human equivalent of LncRNAE330013P06 (E33), which is also named as Cardiac Mesoderm Enhancer-associated Noncoding RNA (CARMN) or MIR143HG [7]. In an animal model study, the LncRNAE33 was found to induce foam cell formation promoting atherosclerosis [8]. And in the same study, LncRNA uc063iod.1 was upregulated in monocytes derived from type II diabetic patients. Study has proven that monocytes play essential roles in initiation, progression and development of atherosclerosis and CAD [9]. E33 is associated with M2 macrophage; and M2 macrophage accelerates the cholesterol uptake and induces foam cell formation promoting atherosclerosis [10]. Intraplaquehemorrhage accelerates atherosclerosis via oxidant stress and contributes to lesion development and destabilization. M2 macrophage is also found in intra plaque hemorrhage in coronary atheroma [11]. LncRNA uc063iod.1 is host gene of miR-143 and miR-145. Several studies suggested that mir-143 and mir-145 is associated with progression development of atherosclerosis and CAD [12] [13] [14] [15]. Therefore, it will be reasonable to speculate that the LncRNA uc063iod.1 may be involved in the progression of CAD.

In this study, the expression pattern of LncRNA uc063iod.1 in plasma of the patients with CAD and a variety of other diseases were detected by qRT-PCR. And we tested the potential correlations between the plasma LncRNA uc063iod.1 levels and clinicopathological features in patients with CAD. The possibility of LncRNA uc063iod.1 to serve as a biomarker for the diagnosis of CAD was discussed. 


\section{Materials and Methods}

\subsection{Study Population}

Plasma samples were prepared using the blood extracted from total 101 patients undergoing coronary artery angiogram (63 CAD patients and 38 Non-CAD patients) in Zhongda Hospital, Southeast University, Nanjing PR China, between May 2017 and October 2017. The study was approved by ethics committee of medical faculty of the Zhongda Hospital, and written informed consent was taken from all included patients or their families in accordance with the Declaration of Helsinki [16].

\subsubsection{Inclusion Criteria}

1) Case: Patients with $>50 \%$ stenosis of at least one of the major coronary arteries (left main coronary trunk, anterior descending branch, circumflex artery and right coronary artery) confirmed by two independent intervention cardiologist during percutaneous coronary artery angiogram.

2) Control: No stenosis in any coronary arteries confirmed by two independent interventional cardiologists.

3) Undergone percutaneous coronary artery angiogram for the first time.

4) Age $>50$ yrs to $<75$ yrs.

\subsubsection{Exclusion Criteria}

1) Patients with severe valvular disease, malignant tumors, other systemic disease like renal failure and hepatic disease.

2) Patients who rejects to take part in the study.

3) Patients with serious acute infections in recent 4 weeks periods.

\subsection{Blood Sample Collection}

$10 \mathrm{ml}$ of arterial blood were collected in EDTA tube from radial artery, after the insertion of the arterial catheter before the administration of anticoagulants. Plasma were collected after centrifugation at $2000 \mathrm{rpm}$ at $4^{\circ} \mathrm{C}$ for $10 \mathrm{mins}$ and stored at $-80^{\circ} \mathrm{C}$ before use.

\subsection{RNA Extraction and $q R T-P C R$}

Total RNA was extracted from plasma using TRIzol reagent (Invitrogen, USA) and cDNA synthesis was carried out by $1 \mu \mathrm{l}$ of RNA, using $5 x$ PrimeScriptRT Master Mix (Takara Biomed Co. Ltd., Beijing) $4 \mu \mathrm{l}$, in total volume of $20 \mu \mathrm{l}$ reaction mixture. SYBR Green Real-Time PCR Master Mixes (Nanjing VazymeBioTech Co. Ltd.) was used to prepare PCR reaction system. The following primers were used in PCR reactions: 5'-TCTTTCTCACAGGCCGCATT-3' (forward) and 5'-TGATTTCTCCACGGTCAGGC-3' (reverse) for LncRNA uc063iod.1; 5'GCACCGTCAAGGCTGAGAAC-3' (forward) and 5'-TGGTGAAGACGCCAGTGGA-3' (reverse) for GAPDH. The primers used were all synthesized by Genscript USA Inc. PCR reaction conditions were: de- 
naturation at $95^{\circ} \mathrm{C}$ for 1 minute, followed by 40 cycles of denaturation $95^{\circ} \mathrm{C}$ for 5 seconds annealing at $60^{\circ} \mathrm{C}$ for 31 seconds. Data were analysed using $2^{-\triangle \Delta C T}$ methods, and relative expression level of LncRNA uc063iod.1 was normalized to endogenous control GAPDH. Each sample was independently tested for three times.

\subsection{Statistical Analysis}

SPSS 20.0 software was used to analyse all data. The test of normality of data was checked by Shapiro-Wilk test. Independent t-test or Mann-Whitney U-test were used to assess the differences in the expression levels of genes between CAD and Non-CAD groups. Continuous variables were tested with student $\mathrm{t}$ test and $2 \times 2$ contingency table analysis of $\chi^{2}$ was used for categorical variables. Abnormally distributed data were processed using non-parametric Mann-Whitney $U$ test. Kruskal-Wallis test was used to test the significance of expression of the gene with respect to the degree of lesion. The correlation between the expression levels of genes was performed by Spearman correlation coefficient. Finally, the Receiver Operating Characteristic (ROC) curve analysis was performed to analyse the sensitivity and specificity of the expression level of LncRNA uc063iod.1. The area under the ROC curve (AUC) was used to summarise the diagnostic accuracy of the genes. A value of $\mathrm{p}<0.05$ was considered statistically significant.

\section{Results}

\subsection{Clinicopathological Characteristics of the Study Population}

Case control study was designed; blood samples were collected from total 101 patients, which were divided into two groups, 63 patients with CADas Case and 38 Non-CAD as Control. Then we conducted a comparison analysis of clinicopathological characteristics of the study population. In this study we found that CAD patients were aged $64.17 \pm 6.60$ years, comparable with $61.88 \pm 3.93$ years in Non-CAD group. There was significance differences between CAD and Non-CAD in status of gender $(\mathrm{p}=0.001)$, hypertension $(\mathrm{p}=0.001), \mathrm{HDL}(\mathrm{p}=$ $0.042)$, WBC $(p=0.048)$, neutrophil $(p=0.027)$, monocyte $(p=0.027)$ and HbAlc $(\mathrm{p}=0.028)$, whereas parameters like age, height, BMI, heart rate, diabetes mellitus II, smoking, trop I, LVEF, lymphocyte, TC, TG and LDL could not show any significance difference (Table 1).

\subsection{LncRNA uc063iod.1 Expression in the Plasma of CAD and Non-CAD Patients}

The expression of LncRNA uc063iod.1 in 63 CAD patients and 38 Non-CAD patients were detected by quantitative real-time polymerase chain reaction. As shown in Figure 1, the expression level of LncRNA uc063iod.1 in plasma of CAD patients were significantly increased compared with the normal control ( $\mathrm{p}$ $<0.01$ ). Hence, our data suggested that the expression of LncRNA uc063iod.1 was upregulated in the plasma of patients of CAD. 
Table 1. Distribution of clinical characteristics in CAD patients and control group.

\begin{tabular}{|c|c|c|c|c|}
\hline Characteristics & & CAD & Non-CAD Control & $p$ value \\
\hline \multirow{5}{*}{$\operatorname{Age}^{\Omega}$ (year) } & $\mathrm{N}$ (Missing) & $63(0)$ & $38(0)$ & \multirow{5}{*}{0.055} \\
\hline & Mean \pm Std. & $64.17 \pm 6.60$ & $61.88 \pm 3.93$ & \\
\hline & $\operatorname{Min} / \operatorname{Max}$ & $52 / 75$ & $54 / 68$ & \\
\hline & Median & 65 & 61.75 & \\
\hline & Range & 23 & 14 & \\
\hline \multirow{3}{*}{ Gender ${ }^{\#}$} & Male & $44(69.84 \%)$ & $14(36.84 \%)$ & \multirow{3}{*}{0.001} \\
\hline & Female & $19(30.16 \%)$ & $24(63.16 \%)$ & \\
\hline & Total (Missing) & $63(0)$ & $38(0)$ & \\
\hline \multirow{5}{*}{ Height $^{\Omega}(\mathrm{cm})$} & $\mathrm{N}$ (Missing) & $63(0)$ & $38(0)$ & \multirow{5}{*}{0.060} \\
\hline & Mean \pm Std. & $167.25 \pm 7.296$ & $164.42 \pm 7.176$ & \\
\hline & $\operatorname{Min} / \operatorname{Max}$ & $147 / 178$ & $148 / 178$ & \\
\hline & Median & 168 & 164 & \\
\hline & Range & 31 & 30 & \\
\hline \multirow{5}{*}{$\mathrm{BMI}^{\Omega}\left(\mathrm{kg} / \mathrm{m}^{2}\right)$} & $\mathrm{N}$ (Missing) & $63(0)$ & $38(0)$ & \multirow{5}{*}{0.974} \\
\hline & Mean \pm Std. & $24.004 \pm 2.587$ & $23.98 \pm 3.17$ & \\
\hline & $\operatorname{Min} / \operatorname{Max}$ & $18.29 / 30.11$ & $17.85 / 32.34$ & \\
\hline & Median & 23.66 & 23.29 & \\
\hline & Range & 11.82 & 14.49 & \\
\hline \multirow{5}{*}{ Heart rate $^{\Omega}(\mathrm{bpm})$} & $\mathrm{N}$ (Missing) & $63(0)$ & $38(0)$ & \multirow{5}{*}{0.415} \\
\hline & Mean \pm Std. & $77.21 \pm 12.75$ & $79.47 \pm 14.64$ & \\
\hline & Min/Max & $60 / 120$ & $51 / 126$ & \\
\hline & Median & 74 & 79 & \\
\hline & Range & 60 & 75 & \\
\hline \multirow{3}{*}{$\begin{array}{l}\text { Hypertension }{ }^{\#} \\
(\mathrm{~mm} \text { of } \mathrm{Hg})\end{array}$} & Yes & $49(77.78 \%)$ & $14(36.84 \%)$ & \multirow{3}{*}{0.001} \\
\hline & No & $14(22.22 \%)$ & $24(63.16 \%)$ & \\
\hline & Total (Missing) & $63(0)$ & $38(0)$ & \\
\hline \multirow{3}{*}{$\begin{array}{l}\text { Diabetes mellitus } \\
\text { type } \mathrm{II}^{\#}\end{array}$} & Yes & $38(60.31 \%)$ & $17(40.74 \%)$ & \multirow{3}{*}{0.128} \\
\hline & No & $25(39.69 \%)$ & $21(55.26 \%)$ & \\
\hline & Total (Missing) & $63(0)$ & $38(0)$ & \\
\hline \multirow{3}{*}{ Smoking $^{*}$} & Yes & $19(30.16 \%)$ & $10(26.32 \%)$ & \multirow{3}{*}{0.679} \\
\hline & No & $44(69.84 \%)$ & $28(73.68 \%)$ & \\
\hline & Total (Missing) & $63(0)$ & $38(0)$ & \\
\hline \multirow{5}{*}{$\operatorname{TropI}^{\varnothing}$ (ng/ml) } & $\mathrm{N}$ (Missing) & $61(2)$ & $36(2)$ & \multirow{5}{*}{0.070} \\
\hline & Mean \pm Std. & $0.706 \pm 3.09$ & $0.14 \pm 0.178$ & \\
\hline & $\operatorname{Min} / \operatorname{Max}$ & $0.1 / 19$ & $00 / 0.1$ & \\
\hline & Median & 0.01 & 0.01 & \\
\hline & Range & 19.01 & 0.1 & \\
\hline
\end{tabular}




\section{Continued}

\begin{tabular}{|c|c|c|c|c|}
\hline & $\mathrm{N}$ (Missing) & $49(14)$ & $24(14)$ & \\
\hline & Mean \pm Std. & $0.706 \pm 3.09$ & $0.672 \pm 3.83$ & \\
\hline \multirow[t]{5}{*}{$\operatorname{HbAlC}^{\varnothing}(\%)$} & Min/Max & $5.20 / 14.30$ & $5.20 / 10.50$ & 0.028 \\
\hline & Median & 7.10 & 6.15 & \\
\hline & Range & 9.10 & 5.30 & \\
\hline & $\mathrm{N}$ (Missing) & $52(11)$ & $33(5)$ & \\
\hline & Mean \pm Std. & $66.25 \pm 7.17$ & $67.57 \pm 6.65$ & \\
\hline \multirow[t]{5}{*}{$\operatorname{LVEF}^{\emptyset}(\%)$} & Min/Max & $52 / 80$ & $55 / 80$ & 0.481 \\
\hline & Median & 67 & 68 & \\
\hline & Range & 28 & 25 & \\
\hline & $\mathrm{N}$ (Missing) & $61(2)$ & $37(1)$ & \\
\hline & Mean \pm Std. & $6.818 \pm 2.268$ & $5.9611 \pm 1.812$ & \\
\hline \multirow[t]{5}{*}{$\mathrm{WBC}^{\emptyset}\left(10^{9} / \mathrm{L}\right)$} & Min/Max & $3.05 / 15.56$ & $3.36 / 13.01$ & 0.048 \\
\hline & Median & 6.51 & 5.6200 & \\
\hline & Range & 12.51 & 9.65 & \\
\hline & $\mathrm{N}$ (Missing) & $61(2)$ & $37(1)$ & \\
\hline & Mean \pm Std. & $4.624 \pm 2.051$ & $3.856 \pm 1.638$ & \\
\hline \multirow{5}{*}{ Neutrophil $^{\varnothing}\left(10^{9} / \mathrm{L}\right)$} & $\operatorname{Min} / \mathrm{Max}$ & $1.63 / 13.26$ & $1.61 / 11.22$ & 0.027 \\
\hline & Median & 4.22 & 3.53 & \\
\hline & Range & 11.63 & 9.61 & \\
\hline & $\mathrm{N}$ (Missing) & $61(2)$ & $37(1)$ & \\
\hline & Mean \pm Std. & $1.633 \pm 0.739$ & $1.635 \pm 0.561$ & \\
\hline \multirow[t]{5}{*}{ Lymphocyte $^{\Omega}\left(10^{9} / \mathrm{L}\right)$} & $\operatorname{Min} / \operatorname{Max}$ & $0.34 / 5.69$ & $0.53 / 2.71$ & 0.986 \\
\hline & Median & 1.47 & 1.62 & \\
\hline & Range & 5.35 & 2.18 & \\
\hline & $\mathrm{N}$ (Missing) & $61(2)$ & $37(1)$ & \\
\hline & Mean \pm Std. & $0.411 \pm 0.171$ & $0.339 \pm 0.126$ & \\
\hline \multirow[t]{5}{*}{ Monocyte $^{\varnothing}\left(10^{9} / \mathrm{L}\right)$} & $\operatorname{Min} / \operatorname{Max}$ & $0.19 / 0.89$ & $0.16 / 0.74$ & 0.027 \\
\hline & Median & 0.40 & 0.31 & \\
\hline & Range & 0.70 & 0.58 & \\
\hline & $\mathrm{N}$ (Missing) & $58(5)$ & $31(7)$ & \\
\hline & Mean \pm Std. & $4.355 \pm 1.225$ & $4.4729 \pm 0.972$ & \\
\hline \multirow[t]{5}{*}{$\mathrm{TC}^{\Omega}(\mathrm{mmol} / \mathrm{L})$} & $\operatorname{Min} / \operatorname{Max}$ & $2.16 / 8.86$ & $2.89 / 6.94$ & 0.646 \\
\hline & Median & 4.25 & 4.57 & \\
\hline & Range & 6.70 & 4.05 & \\
\hline & $\mathrm{N}$ (Missing) & $58(5)$ & $31(7)$ & \\
\hline & Mean \pm Std. & $1.768 \pm 0.0677$ & $1.554 \pm 1.0732$ & \\
\hline \multirow[t]{3}{*}{$\mathrm{TG}^{\varnothing}(\mathrm{mmol} / \mathrm{L})$} & $\operatorname{Min} / \operatorname{Max}$ & $0.7 / 4.9$ & $0.5 / 4.9$ & 0.288 \\
\hline & Median & 1.355 & 1.230 & \\
\hline & Range & 4.3 & 4.3 & \\
\hline
\end{tabular}




\section{Continued}

\begin{tabular}{ccccc}
\hline & N (Missing) & $58(5)$ & $31(7)$ & \\
& Mean \pm Std. & $1.088 \pm 0.242$ & $1.200 \pm 0.25$ & \\
$\mathrm{HDL}^{\Omega}(\mathrm{mmol} / \mathrm{L})$ & Min/Max & $0.75 / 1.88$ & $0.81 / 1.60$ & 0.042 \\
& Median & 1.055 & 1.20 & \\
Range & 1.13 & 0.79 & \\
& N (Missing) & $58(5)$ & $31(7)$ & \\
& Mean \pm Std. & $3.093 \pm 1.259$ & $2.58 \pm 0.738$ & \\
& Min/Max & $1.10 / 6.52$ & 1.41 & \\
$\mathrm{LDL}^{\Omega}(\mathrm{mmol} / \mathrm{L})$ & Median & 2.81 & 4.24 & \\
& Range & 5.42 & 2.83 & \\
\hline
\end{tabular}

$\mathrm{BMI}=$ Body mass index, $\mathrm{bpm}=$ beats per minute, $\mathrm{TG}=$ triglyceride, $\mathrm{TC}=$ total cholesterol, $\mathrm{HDL}=$ high density lipoprotein, $\mathrm{LDL}=$ low density lipoprotein, $\mathrm{WBC}=$ white blood cell, $\mathrm{LVEF}=$ left ventricular ejection fraction, TropI $=$ troponin $\mathrm{I}, \mathrm{HbAlc}=$ glycated haemoglobin. Data are presented as mean \pm standard deviation (std.), Minimum/Maximum (Min/Max), Median and Range. $\mathrm{N}=$ number of patients. $\mathrm{P}<0.05$ was considered significant. ${ }^{\Omega}$ Student T-test. ${ }^{\#} 2 \times 2$ contingency table analysis of $\chi 2 .{ }^{\emptyset} \mathrm{Mann}$-Whitney U-test.

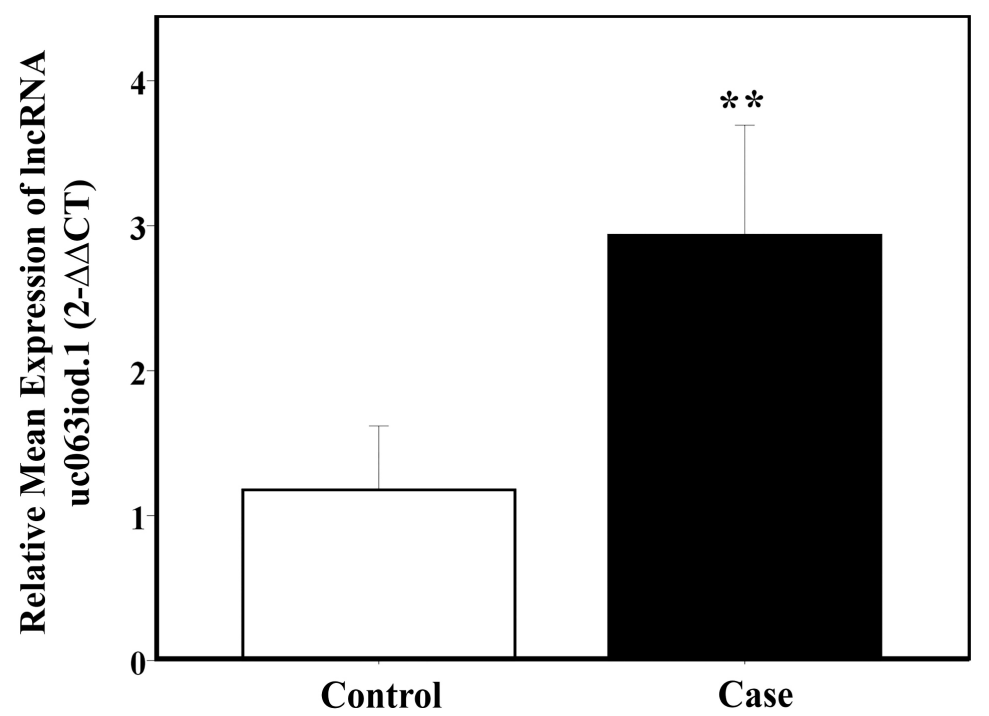

Figure 1. The relative mean expression of LncRNA uc063iod.1 in the plasma of CAD patients and Non-CAD control. ${ }^{* *}$ compared with control group, $\mathrm{p}<0.01$.

\subsection{Expression of LncRNA uc063iod.1 in Patients with Respect to the Coronary Artery Lesion}

The mean expression level LncRNA uc063iod.1 in plasma of CAD patients found to be statistically significant with respect to numbers of coronary arteries involved, i.e. $\mathrm{p}<0.01$. The LncRNA uc063iod.1 expression is significantly increased in single vessel, bi vessel and three vessel disease than in non CAD patients. But there was no significant difference between the expression level of LncRNA uc063iod.1 with respect to degree of lesion in coronary arteries among CAD patients (Figure 2). 


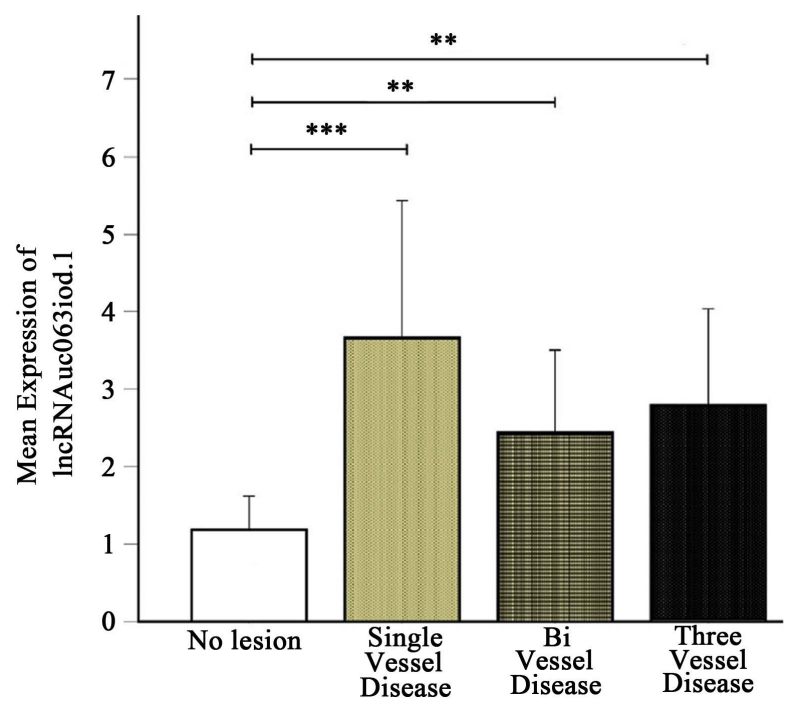

Figure 2. The mean expression of LncRNA uc063iod.1 in plasma of CAD patients with respect to diseased number of coronary arteries and the control with no lesion. ${ }^{* *} \mathrm{p}<0.01$ and ${ }^{* * *} \mathrm{p}<0.001$.

\subsection{Expression of LncRNA uc063iod.1 in CAD Patients with Stable Angina Pectoris, Unstable Angina Pectoris and Acute Myocardial Infarction}

Plasma levels of LncRNA uc063iod.1 in patients with Stable Angina Pectoris, unstable Angina Pectoris and Acute Myocardial Infarction found to have significant difference with the control $(\mathrm{p}<0.01)$. As shown in Figure 3, patients presented with SAP and AMI appeared to have significantly higher levels of LncRNA uc063iod.1, although there was no significant difference observed among these three sub-groups (Figure 3).

\subsection{Correlation Ship between LncRNA uc063iod.1 and Coromarker}

Coromarker is already an established biomarker of CAD [17]. A case control study done by Yue et al. has claimed Coromarker to be highly sensitive and specific for the diagnosis of CAD. They also validated it for its diagnostic accuracy in a prospective way. They found that patients with overly expressed plasma Coromarker were later diagnosed with CAD during coronary artery angiogram. In this study we also evaluate the expression of Coromaker, and the result was consistent with their findings, i.e. Coromarker is overly expressed in plasma of CAD patients, $\mathrm{p}<0.05$. We also had bivariate spearman correlation analysis between LncRNA uc063iod.1 and Coromarker which showed the expression of LncRNA uc063iod.1 in plasma was positively correlated with the expression of Coromarker the correlation coefficient $r=0.224$ and $p=0.024$, shown in Table 2 .

\subsection{Correlation Ship of genes with Some Clinical Features of CAD and Non CAD Patients}

The correlation ship of the genes with clinical features of CAD and Non CAD 


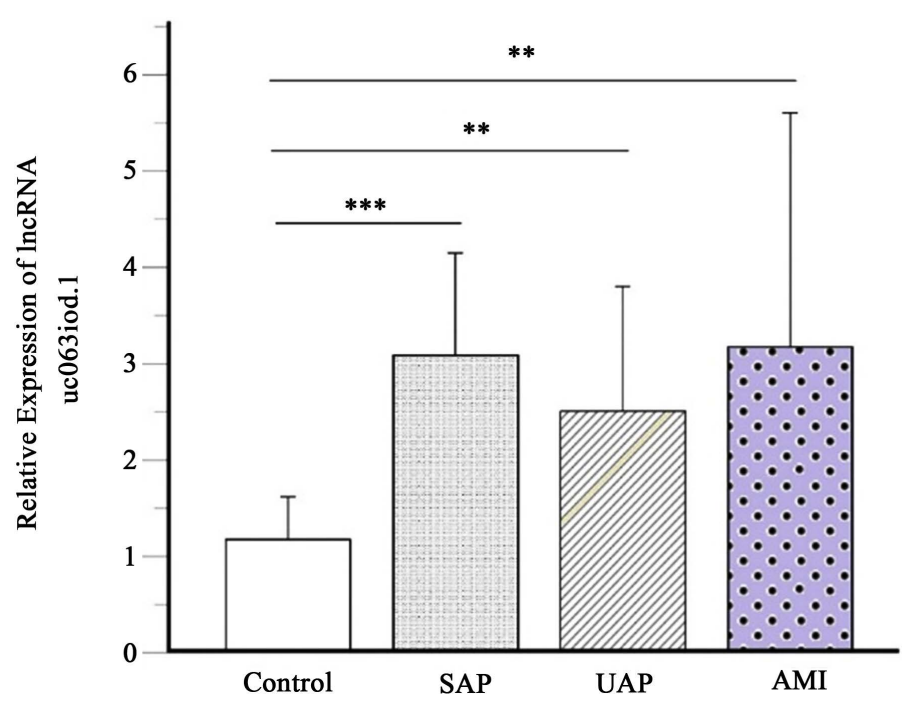

Figure 3. The relative expression of LncRNA uc063iod.1 in plasma of CAD patients showed significant difference with control group, ${ }^{* *} \mathrm{p}<0.01$ and ${ }^{\star * *} \mathrm{p}<0.001$. SAP $=$ Stable Angina Pectoris, UAP $=$ Unstable Angina Pectoris, AMI = Acute Myocardial Infarction.

Table 2. Correlation ship between LncRNA uc063iod.1 and Coromarker.

\begin{tabular}{ccc}
\hline & Coromarker & \\
LncRNA uc063iod.1 & $\mathrm{r}$ & p value \\
& 0.225 & 0.024 \\
\hline
\end{tabular}

patients were calculated by bivariate spearman correlation analysis, which showed positive correlation ship between the expression levels of LncRNA uc063iod.1 with DM type II ( $\mathrm{r}=0.327, \mathrm{p}=0.009)$, hypertension $(\mathrm{r}=0.296, \mathrm{p}=$ $0.018)$ and negative correlation ship with Smoking $(r=-0.363, p=0.003)$ in CAD patients. There was negative correlation ship between the expression levels of LncRNA uc063iod. 1 with diabetes mellitus II $(r=-0.437, \mathrm{p}=0.006)$, hypertension $(r=0.413, p=0.01)$ among Non CAD patients, shown in Table 3. There were no significant correlation ship establish on status of TC, TG, HDL, LDL, HbAlc.

\subsection{Plasma LncRNA uc063iod.1 Is Sensitive for CAD}

Receiver Operating Characteristic (ROC) curve was used to determine the best positive plasma LncRNA uc063iod.1 reference values to improve its diagnostic value of CAD. The area under the ROC curve (AUC) of LncRNA uc063iod.1 for diagnosing CAD was 0.701 (95\%CI: 0.598 - 0.804), with the sensitivity $77.8 \%$ and specificity $57.9 \%$ (Figure $4(\mathrm{a})$ ). In our study ROC curve analysis of the expression of Coromarker for diagnosing CAD was 0.645 (95\% CI: 0.53 - 0.752), with the sensitivity of $60.3 \%$ and the specificity of $73.7 \%$ (Figure $4(\mathrm{~b})$ ). This suggested that upregulation of LncRNA uc063iod. 1 in plasma can be used as a biomarker for the diagnosis of CAD. 


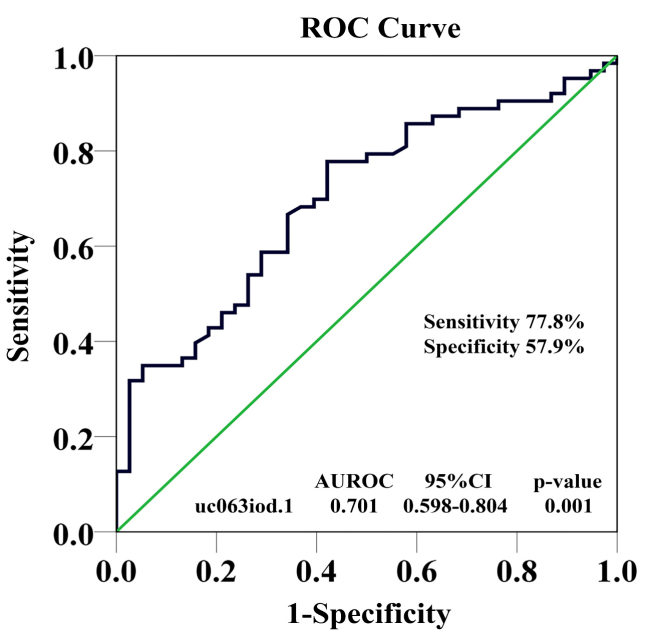

(a)

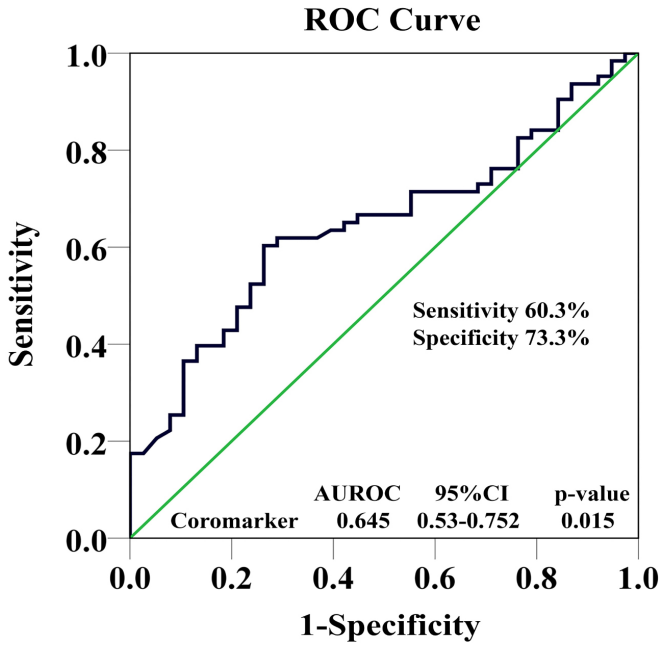

(b)

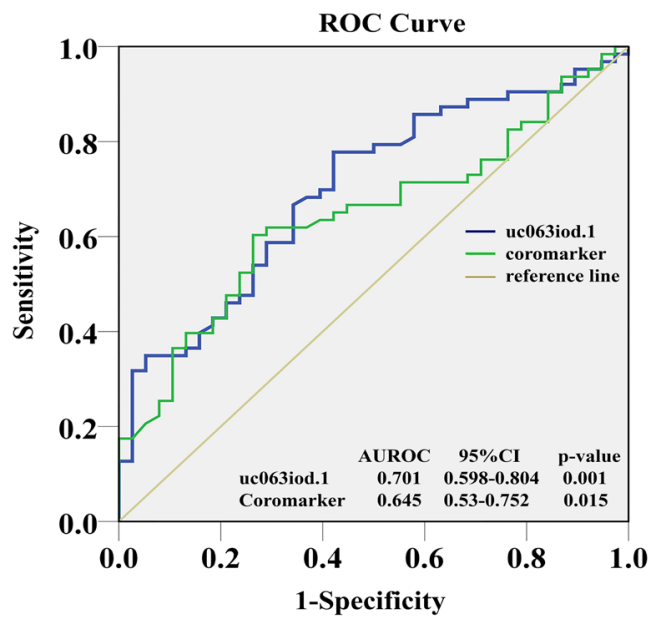

(c)

Figure 4. ROC curve analysis of LncRNA LncRNA uc063iod.1 and Coromarker: (Figures (a)-(c)) AUC of LncRNA uc063iod.1 was 0.701 (95\%CI: 0.598 - 0.804), with the sensitivity $77.8 \%$ and specificity $57.9 \%, \mathrm{p}=0.001$. AUC of Coromarker was 0.645 (95\% CI: 0.53 0.752 ), with the sensitivity of $60.3 \%$ and specificity of $73.7 \%, \mathrm{p}=0.015$. 
Table 3. Correlation ship of genes with some clinical features of CAD and Non CAD patients.

\begin{tabular}{cccccc}
\hline & $\begin{array}{c}\text { Correlation } \\
\text { with }\end{array}$ & \multicolumn{2}{c}{ CAD } & \multicolumn{2}{c}{ Non CAD control } \\
& Smoking & -0.363 & $\mathbf{0 . 0 0 3}$ & 0.076 & 0.649 \\
LncRNA & DM II & 0.327 & $\mathbf{0 . 0 0 9}$ & -0.437 & $\mathbf{0 . 0 0 6}$ \\
uc063iod.1 & Hypertension & 0.296 & $\mathbf{0 . 0 1 8}$ & -0.413 & $\mathbf{0 . 0 1}$ \\
& TC & 0.202 & 0.129 & -0.188 & 0.311 \\
& TG & 0.039 & 0.769 & -0.306 & 0.094 \\
& HDL & 0.027 & 0.841 & 0.015 & 0.937 \\
& LDL & 0.156 & 0.240 & -0.096 & 0.606 \\
& HbAlc & -0.08 & 0.586 & -0.077 & 0.720 \\
\hline & Smoking & 0.074 & 0.563 & 0.005 & 0.974 \\
& DM II & 0.131 & 0.306 & -0.036 & 0.829 \\
& Hypertension & 0.277 & $\mathbf{0 . 0 2 8}$ & -0.020 & 0.906 \\
& TC & -0.20 & 0.880 & -0.355 & $\mathbf{0 . 0 5 0}$ \\
& TG & 0.337 & $\mathbf{0 . 0 1}$ & -0.374 & $\mathbf{0 . 0 3 8}$ \\
& HDL & -0.109 & 0.415 & -0.136 & 0.465 \\
& LDL & 0.234 & 0.77 & -0.311 & 0.088 \\
& HbAlc & 0.081 & 0.580 & -0.224 & 0.293 \\
\hline
\end{tabular}

DM II = Diabetes Mellitus type II, TG = triglyceride, $\mathrm{TC}=$ total cholesterol, $\mathrm{HDL}=$ high density lipoprotein, $\mathrm{LDL}=$ low density lipoprotein, $\mathrm{HbAlc}=$ glycatedhemoglobin.

\section{Discussion}

Coronary Artery Disease (CAD) is major cause of sudden cardiac death and it usually progresses to myocardial infarction if remain untreated. Among the individuals with CVDs, coronary artery disease accounts for $22 \%$ of early deaths and 15\% of late death [18]. Various studies have pointed several LncRNAs to be involved in development of coronary artery disease. Genome-wide association studies have found that regions on chromosome 9p21 (Chr9p21) are associated with various diseases including $\mathrm{CAD}$ and other coronary heart diseases [19]. The LncRNA antisense noncoding RNA in the INK4 locus (ANRIL) found on human chromosome 9p21, was the first LncRNA proven to be able to contribute to the susceptibility of CAD [20]. The altered expression of LncRNAANRIL on chromosome 9p21 mediates methylation of p15INK4b and regulates inflammatory responses as a novel component of NF-kB pathway which is associated with the development of CAD [21] [22]. MAPK and NF-kB signalling pathways have been reported to be a central mediator and a key participant of the inflammatory process, and also involved in regulation of atherosclerosis [23]. J-K PAN found that LncRNAH19 regulates atherosclerosis by activating the MAPK and NF-kB signalling pathway [24]. Recent study claimed LncRNAZFAS1 and LncRNACDR1AS 
showed significant differences in their circulating level between Acute Myocardial Infarction (AMI) patients and control subjects and they found that the expression of LncRNAZFAS1 is reduced and expression of LncRNACDR1AS is increased in AMI [25]. In another study by R. Kumaraswamy et al., found that LncRNA uc022bqs.1 was overly expressed in heart failure patients following AMI incident. And they discovered the LncRNA uc022bqs. 1 can predict the survival in patients with heart failure [5]. Therefore, it can be suggested that LncRNAs are closely associated with the development of Coronary Heart Diseases; from atherosclerotic cardiovascular diseases (ASCVD) to chronic heart failure. Therefore the LncRNAs have potential to be a great breakthrough in diagnosis and management of cardiovascular diseases.

LncRNA uc063iod.1 being host gene of mir-143 and mir-145, is a crucial regulator of cardiac cell differentiation and homeostasis [26]. In our study we found that the expression of plasma LncRNA uc063iod.1 in CAD patients is upregulated than the Non CAD patients. A ROC curve was analyzed for distinguishing CAD patients from the Non CAD patients, and the results demonstrated that the AUC was $0.701,(95 \% \mathrm{CI}: 0.598$ - 0.808), and the sensitivity and specificity were $77.8 \%$ and $57.9 \%$ respectively. The result strongly suggests towards the feasibility of the LncRNA uc063iod.1 as biomarker for CAD. We found significance differences between CAD and Non-CAD in status of gender, hypertension, HDL, WBC, neutrophil, monocyte and HbAlc. We further studied the association of the expression level with the number of coronary arteries involved and we found significant association between expression of LncRNA uc063iod.1 and degree of lesions among CAD and Non CAD group. But we could not find any significant relationship with the degree of lesions among CAD patients. We also found significant higher level of expression of LncRNA uc063iod.1 instable angina pectoris, unstable angina pectoris and acute myocardial infarction patients than Non CAD patients. This result suggests that there is no varying in levels of the lncRNA uc063iod.1 and the severity of Coronary Artery Disease.

Coromarker is already an established biomarker of CAD. We had bivariate spearman correlation analysis between LncRNA uc063iod.1 and Coromarker which showed positive correlation. This result helps to provide supporting point for LncRNA uc063iod.1 to serve as biomarker for Coronary Artery Disease. Furthermore, we observed the positive correlation ship between the expression level of LncRNA uc063iod.1 with smoking, hypertension and diabetes mellitus type II in CAD patients, whereas negative correlation between the level of LncRNA uc063iod.1 with hypertension and diabetes mellitus in Non CAD patients. The underlying mechanism of these correlation is still unknown. However, there is a study indicated that the upregulation of the level of LncRNA uc063iod.1 in monocytes derived from diabetic patients [8], which in part may explain the positive correlation ship of LncRNA uc063iod. 1 and diabetes in CAD patients. But at the same time it fails to explain the negative correlation with 
diabetes in Non CAD patients. The LncRNA uc063iod.1 is host gene of mir-143 and mir-145. And miR-143/-145 in human peripheral blood mononuclear cells have been found to be upregulated in patients with essential hypertension [27]. The positive correlation ship between the level of LncRNA uc063iod.1 and hypertension in CAD patients may be explain by linking up with this study, but the negative correlation between the level of LncRNA uc063iod.1 and hypertension in Non CAD patients cannot be explained on the basis of this study. So the exact mechanism underlying for these correlations cannot be explained and need further study. We were not able to establish any statistical significant correlation ship between plasma expression level of LncRNA uc063iod.1 with HbA1c and lipids among CAD patients. And it should be noted that all these findings are very preliminary in view of low sample size.

\section{Limitation and Conclusion}

The study was conducted in a Chinese Han Population who underwent coronary artery angiogram in Zhongda Hospital, Nanjing, China. Further studies expanded to different population groups and regions may ensure the true significance of the association between LncRNA uc063iod.1 and CAD. Selection bias might have affected our results. The sample size is relatively very small. Therefore, multicentre large number of studies are needed to be conducted for validation and consideration of LncRNA uc063iod.1 as biomarker of CAD. In conclusion, the expression for LncRNA uc063iod.1 was upregulated in the plasma of patients with CAD. However, the mechanism and pathway of the LncRNA uc063iod.1 in progression of CAD need further exploration.

\section{Conflict of Interest}

The authors declare that there is no conflict of interest.

\section{References}

[1] (2017) WHO Fact Sheet, Cardiovascular Disease.

[2] Ponting, C.P., Oliver, P.L. and Reik, W. (2009) Evolution and Functions of Long Noncoding RNAs. Cell, 136, 629-641. https://doi.org/10.1016/j.cell.2009.02.006

[3] Verhaegh, G.W., et al. (2003) DD3, a Very Sensitive and Specific Marker to Detect Prostate Tumors. Cancer Research, 62, 2695-2698.

[4] Ma, J.X., Cui, X.Q., Rong, Y., Zhou, Y., Guo, Y., Zhou, M., Xiao, L. and Chen, W.H. (2016) Plasma, LncRNA-ATB, a Potential Biomarker for Diagnosis of Patients with Coal Workers' Pneumoconiosis: A Case-Control Study. International Journal of Molecular Sciences, 17, 1367. https://doi.org/10.3390/ijms17081367

[5] Kumarswamy, R., Bauters, C., Volkmann, I., et al. (2014) Circulating Long Noncoding RNA, LIPCAR, Predicts Survival in Patients with Heart Failure. Circulation Research, 114, 1569-1575. https://doi.org/10.1161/CIRCRESAHA.114.303915

[6] Vaidya Rajan, M.G.S., Sabina, S., Naresh, K., Jasmine, S. and Farhan, K. (2018) Long Non-Coding RNAs and Coronary Artery Disease. International Journal of Science Inventions Today, 7, 80-88. 
[7] HUGO Gene Nomeclature Committe.

[8] Reddy, M., et al. (2014) Regulation of Inflammatory Phenotype in Macrophages by a Diabetes-Induced Long Noncoding RNA. 63.

[9] Ghattas, A., et al. (2013) Monocytes in Coronary Artery Disease and Atherosclerosis: Where Are We Now? Journal of the American College of Cardiology, 62, 1541-1551. https://doi.org/10.1016/j.jacc.2013.07.043

[10] Oh, J., et al. (2012) Endoplasmic Reticulum Stress Controls M2 Macrophage Differentiation and Foam Cell Formation. 287, 11629-11641.

[11] Boyle, J.J., et al. (2009) Coronary Intraplaque Hemorrhage Evokes a Novel Atheroprotective Macrophage Phenotype. American Journal of Pathology, 174, 1097-1108.

[12] Gao, H., Guddeti, R.R., Matsuzawa, Y., Liu, L.-P., Su, L.-X., Guo, D., et al. (2015) Plasma Levels of microRNA-145 Are Associated with Severity of Coronary Artery Disease. PLoS ONE, 10, e0123477. https://doi.org/10.1371/journal.pone.0130780

[13] Shrestha, S., Ren, L. and Vaidya, R. (2018) miRNAs as Biomarkers for Diagnosis and Assessment of Prognosis of Coronary Artery Disease. Open Journal of Internal Medicine, 8, 10.

[14] Sala, F., et al. (2014) MiR-143/145 Deficiency Attenuates the Progression of Atherosclerosis in Ldlr-/-Mice. Thrombosis and Haemostasis, 112, 796-802.

[15] D'alessandra, Y., et al. (2013) Diagnostic Potential of Plasmatic MicroRNA Signatures in Stable and Unstable Angina. PLOS ONE, 8, e80345.

[16] World Medical Association (2013) World Medical Association Declaration of Helsinki: Ethical Principles for Medical Research Involving Human Subjects. JAMA, 310, 2191-2194. https://doi.org/10.1001/jama.2013.281053

[17] Cai, Y., et al. (2016) Circulating "IncRNA OTTHUMT00000387022" from Monocytes as a Novel Biomarker for Coronary Artery Disease. Cardiovascular Research, 112, 714-724.

[18] Shemirani, H. and Nayeri-Torshizi, E. (2015) Electrocardiographic Characteristics of Posterior Myocardial Infarction in Comparison to Angiographic Findings. ARYA Atherosclerosis, 11, 30-35.

[19] McPherson, R., et al. (2007) A Common Allele on Chromosome 9 Associated with Coronary Heart Disease. Science, 316, 1488-1491. https://doi.org/10.1126/science.1142447

[20] Helgadottir, A., et al. (2007) A Common Variant on Chromosome 9p21 Affects the Risk of Myocardial Infarction. Science, 316, 1491-1493. https://doi.org/10.1126/science.1142842

[21] Zhuang, J., et al. (2012) Methylation of p15INK4b and Expression of ANRIL on Chromosome 9p21 Are Associated with Coronary Artery Disease. PLoS ONE, 7, e47193. https://doi.org/10.1371/journal.pone.0047193

[22] Zhou, X., et al. (2016) Long Non-Coding RNA ANRIL Regulates Inflammatory Responses as a Novel Component of NF- $\kappa$ B Pathway. RNA Biology, 13, 98-108. https://doi.org/10.1080/15476286.2015.1122164

[23] Lee, K., et al. (2016) Inhibition of VCAM-1 Expression on Mouse Vascular Smooth Muscle Cells by Lobastin via Downregulation of p38, ERK $1 / 2$ and NF- $\kappa$ B Signaling Pathways. Archives of Pharmacal Research, 39, 83-93. https://doi.org/10.1007/s12272-015-0687-3

[24] Pan, J.-X. (2017) LncRNA H19 Promotes Atherosclerosis by Regulating MAPK and NF- $\kappa$ B Signaling Pathway. European Review for Medical and Pharmacological Sciences, 21, 322-328. 
[25] Zhang, Y., et al. (2016) Reciprocal Changes of Circulating Long Non-Coding RNAs ZFAS1 and CDR1AS Predict Acute Myocardial Infarction. Scientific Reports, 6, Article No. 22384.

[26] Ounzain, S., et al. (2015) CARMEN, a Human Super Enhancer-Associated Long Noncoding RNA Controlling Cardiac Specification, Differentiation and Homeostasis. Journal of Molecular and Cellular Cardiology, 89, 98-112.

[27] Kontaraki, J., et al. (2013) Differential Expression of Vascular Smooth Muscle-Modulating microRNAs in Human Peripheral Blood Mononuclear Cells: Novel Targets in Essential Hypertension. Journal of Human Hypertension, 28, 510-516. 